\title{
Non-classical vibration behavior of highly anisotropic corrugated laminates
}

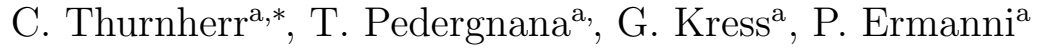 \\ ${ }^{a}$ Laboratory of Composite Materials and Adaptive Structures, Department of Mechanical \\ and Process Engineering, ETH Zürich, Tannenstr. 3, CH-8092 Zürich, Switzerland
}

\begin{abstract}
This paper presents a detailed investigation about the vibration behavior of corrugated laminates. In highly anisotropic corrugated laminates different non-classical vibration modes were observed and are reported in this work. Apart from in-plane modes, we show in particular shear rotational modes which occur due to the high anisotropy, the distribution of mass, and the influence of the shear compliance. The work contains a detailed FEM study, a comparison with an equivalent plate model and an analytical model and examines the limitations of the latter two. It points out for which geometry and material parameters the well known and often used homogenized plate models are applicable. Parametric studies are conducted investigating the influence of the corrugation amplitude, the aspect ratio, the anisotropy of the material, and boundary conditions on the vibration behavior. The found results can be used for the design of highly anisotropic corrugated laminated plates and the analysis of their vibration behavior.
\end{abstract}

Keywords: corrugated laminates, vibration, non-classical effects, flexible

\footnotetext{
${ }^{*}$ Corresponding author

Email address: thclaudi@ethz.ch (C. Thurnherr)
} 
skins, anisotropy 


\section{Introduction}

Corrugated laminates have a very high anisotropy, not only because of the anisotropic laminated materials, but mainly due to geometric reasons. This makes them interesting as structural elements in various applications [1]. They have been used since decades as stability elements, e.g. in Junkers Ju-52 [2] and are widely used as sandwich cores [3, 4]. Lately, they have been suggested as ideal candidates for morphing skins $[5,6]$, for example in morphing wing applications $[7,8]$. In morphing wings flaps and slats are replaced by shape adaptive wings which requires flexible elements such as corrugated laminates that provide axial compliance in order to keep actuation forces low while providing high bending stiffness to withstand external forces. Morphing wing solutions have been suggested as being more efficient than conventional wings $[9]$.

In order to reduce numerical costs, many models have been developed to find the equivalent stiffness properties of corrugated laminates. Xia et al. suggested an analytical model for symmetric and balanced laminates [10, 11]. Kress et al. proposed an analytical model that is valid for corrugations consisting of circular sections [12].

Corrugated laminates with high amplitudes can be favorable due to their stress distribution [13], and enhanced anisotropic behavior. This high anisotropy can lead to in-plane eigenmodes of natural vibrations. Wang et al. [14] also reported in-plane modes in highly anisotropic sandwich structures where the shear compliance is of importance. While the out-of-plane vibration behavior of orthotropic plates is already understood well [15], not much literature about in-plane vibration of highly anisotropic plates is available. Bardell 
et al. [16] analyzed free in-plane vibrations in isotropic plates. Semenyuk et al. [17] investigated different vibration modes of corrugated cylindrical shells. Liu et al. [18] developed an analytical model to analyze in-plane vibration modes for orthotropic rectangular plates. However, non-classical vibration modes of corrugated laminates with extremely high anisotropy where in comparison to flat plates also through-thickness mass distribution has to be accounted for - have not been reported so far in literature. These non-classical vibration modes also limit the valid solution space of often used equivalent plate models. To the authors' knowledge also the question of how anisotropy due to geometry and material influence the observed mode shapes and frequencies of corrugated laminates, which is of high importance for design aspects, have not been addressed so far.

In the present paper we investigate the influence of anisotropy on the vibration behavior of corrugated laminates. We use a detailed FEM model to investigate the vibration behavior as a function of anisotropy due to geometry. Besides the intuitive out-of-plane mode known from anisotropic plate vibration models, we also report in-plane modes and non-classical shear rotational modes. We compare these results with a numerical and an analytical homogenized plate models which are very efficient in terms of numerical costs. Both models use equivalent stiffness properties as input parameters. Comparing the three models allows to quantify the valid solution space for the widely used homogenized plate models which are not able to capture all the observed inertia effects. Furthermore, we investigate the interaction of anisotropy due to material and geometry and their influence on corrugated laminates vibration behavior using analytical modeling. In a parametric 
study the main influencing parameters such as corrugation amplitude, plate aspect ratio, and material properties are examined concerning eigenmodes and -frequencies.

The following section of the paper presents the detailed FEM shell-model, an equivalent homogenized plate FEM model, and an analytical homogenized model. Then the characteristically observed eigenmodes are presented depending on the anisotropy due to geometry. These include out-of-plane, in-plane and rotational modes. The subsequent section investigates the valid solution space for homogenized modeling approaches. Then we add a further complexity by considering the anisotropy due to material and investigate the influence on the vibration modes. Furthermore, a parametric study is presented examining the influence of geometry, material, and aspect ratio on the eigenmodes and frequencies. The paper closes with a discussion of the results and a conclusion.

\section{Modeling approach}

For the modeling of the eigenvalues three different approaches are used:

- an equivalent plate model: efficient and widely used for modeling of corrugated laminates, valid for arbitrary boundary conditions,

- a finite element shell model: allows to accurately map the geometry, valid for arbitrary boundary conditions, but more costly than the other models, and

- an analytical model: very efficient, but only valid for simply-supported boundary conditions. 
Using three different models allows to compare the different results to each other and can be used as a verification in case that all models account for the same assumptions and simplifications. The following subsections describe these models.

\subsubsection{Geometry definition}

Figure 1 shows a unit-cell of a corrugated structure consisting of circular sections as we use it in this work. The corrugation is defined by a corrugation amplitude $c$, a unit-cell length $p$, and a laminate thickness $t_{l a m}$. By changing these parameters various corrugation geometries can be realized. The radius $R$ and the opening angle $\psi_{0}$ can be calculated from the amplitude and the unit-cell length as follows [12]:

$$
R=\frac{16(c / p)^{2}+1}{32 c / p} .
$$

and

$$
\psi_{0}=\left\{\begin{array}{ll}
\operatorname{asin}\left(\frac{p}{4 R}\right), & c \leq \frac{p}{4} \\
\operatorname{acos}\left(\frac{p}{4 R}\right)+\frac{\pi}{2}, & c>\frac{p}{4}
\end{array} .\right.
$$

Figure 1: Definition of geometry of the corrugated structures

Figure 2 shows the geometry of the entire corrugated plate with a size

of $a$ times $b$. All the considered models consist of ten unit-cells or more to ensure that the panel rather than the unit-cell behavior is investigated.

To describe structural stiffness properties of flat laminates we use the following notation of an ABD-matrix as it can also be found in [19]. 
Figure 2: Definition of the corrugated plate geometry

$$
\left[\begin{array}{l}
N \\
M
\end{array}\right]=\left[\begin{array}{ll}
A & B \\
B & D
\end{array}\right]\left[\begin{array}{l}
\epsilon \\
\kappa
\end{array}\right]
$$

while for the equivalent stiffness properties of the corrugated laminate we use the superscript ${ }^{\sim}$ :

$$
\left[\begin{array}{l}
N \\
M
\end{array}\right]=\left[\begin{array}{cc}
\tilde{A} & \tilde{B} \\
\tilde{B} & \tilde{D}
\end{array}\right]\left[\begin{array}{l}
\epsilon \\
\kappa
\end{array}\right] .
$$

\subsection{Equivalent plate model}

Very often corrugated laminates are modeled using homogenized equivalent properties depending on the corrugation geometry and material. These properties can then be used in analytical or numerical models to calculate the structural response of the inner domain solution. In the present study we use the model by Kress and Winkler [12] to calculate the equivalent plate properties of the corrugated structure. The model is valid for thin corrugations consisting of circular sections and cross-ply lay-ups. The equivalent properties are then used in a pre-integrated flat finite element shell model to calculate the vibration behavior of a finite corrugated plate with various boundary conditions. The model is implemented in the commercial FEM code ANSYS using the SHELL181 element (a 4 node shell element).

The advantage of this model is its numerical efficiency. In comparison to a detailed model it can only model homogenized structural responses. It is slightly more costly than an analytical model, but it can be used for any 
kind of boundary conditions and it considers shear stiffnesses $\tilde{K}_{44}$ and $\tilde{K}_{55}$ which couple transverse shear line loads with transverse shear strains. The values are set to $\tilde{K}_{44}=10^{4} \mathrm{~N} / \mathrm{mm}$ and $\tilde{K}_{55}=1 \mathrm{~N} / \mathrm{mm}$ for $c / p>0.25$ and $\tilde{K}_{44}=5000 \mathrm{~N} / \mathrm{mm}$ and $\tilde{K}_{55}=1000 \mathrm{~N} / \mathrm{mm}$, respectively, for $c / p \leq 0.25$ based on considerations made in [20].

\subsection{Finite element shell model}

The second model that is used is a finite element shell model where the geometry is modeled in detail. Figure 3 shows an example of this model with an aspect ratio $a / b=2$ and an amplitude to period cell-length ratio of $c / p=0.5$. The model is also implemented in ANSYS. It uses a four node pre-integrated shell element $S H E L L 181$, but instead of using the equivalent corrugated properties it uses the properties of the sheet $A B D$-matrix. This model is numerically more costly than equivalent models, but it has the advantage that it can calculate global as well as local phenomena.

Figure 3: Detailed finite element shell model

\subsection{Analytical model}

Two analytical models are used to model out-of-plane and in-plane vibration behavior, respectively. Both models are restricted to simply-supported boundary conditions on all four edges and use equivalent stiffness properties as input parameters. 


\section{Out-of-plane mode}

The first out-of-plane natural eigenfrequency $w_{11}^{o o p}$ of an orthotropic plate that is simply supported at all four edges can be calculated as presented by Reddy [15]:

$$
\left(\omega_{11}^{o o p}\right)^{2}=\frac{\pi^{4}}{\rho_{e q} t_{l a m} a^{4}}\left(\tilde{D}_{11}\left(\frac{a}{b}\right)^{4}+2\left(\tilde{D}_{12}+2 \tilde{D}_{66}\right)\left(\frac{a}{b}\right)^{2}+\tilde{D}_{22}\right)
$$

where $\rho_{e q}$ is the equivalent density of the corrugated plate calculated as follows:

$$
\rho_{e q}=\rho \frac{4 \psi_{0} R}{p}
$$

\section{In-plane mode}

The analytical model to calculate the in-plane natural frequency value can be adopted from [18].

The first in-plane natural frequency $w_{11}^{i p}$ can be written as:

$$
\begin{aligned}
\left(\omega_{11}^{i p}\right)^{2} & =\frac{\pi^{2} \tilde{A}_{66}}{2 \rho_{e q} t_{l a m} a^{2}}\left(\left(1+a_{1}\right)\left(\frac{a}{b}\right)^{2}+\left(1+a_{2}\right)\right. \\
& \left.-\sqrt{\left(\left(1-a_{1}\right)\left(\frac{a}{b}\right)^{2}-\left(1-a_{2}\right)\right)^{2}+4 a_{3}^{2}\left(\frac{a}{b}\right)^{2}}\right)
\end{aligned}
$$

where

$$
a_{1}=\frac{\tilde{A}_{11}}{\tilde{A}_{66}}, a_{2}=\frac{\tilde{A}_{22}}{\tilde{A}_{66}}, a_{3}=\frac{\tilde{A}_{12}+\tilde{A}_{66}}{\tilde{A}_{66}} .
$$

Determination of first mode shape

To predict whether an out-of-plane or an in-plane mode is detected the following criterion can be applied: 


$$
f_{\text {equ }}^{\text {analytical }}=\frac{1}{2 \pi} \begin{cases}\omega_{11}^{i p}, & \omega_{11}^{i p}<\omega_{11}^{o o p} \\ \omega_{11}^{o o p}, & \omega_{11}^{i p} \geq \omega_{11}^{o o p}\end{cases}
$$

\subsection{Boundary conditions}

In the present work we focus on two sets of different boundary conditions. In one case all the four edges are simply-supported (thus all the three displacement components are set to zero at $z=0$ at the edges), in the second case all the four edges are clamped (thus all the three displacement and rotation components are set to zero at the edges). The numerical models can be used for both cases while the analytical model is only valid for simplysupported boundary conditions.

\subsection{Material}

For the current study we use a carbon fiber epoxy composite with the properties as listed in table 1.

\begin{tabular}{|l|l|l|l|l|l|}
\hline$E_{11}[\mathrm{GPa}]$ & 135 & $E_{22}[\mathrm{GPa}]$ & 10 & $E_{33}[\mathrm{GPa}]$ & 10 \\
\hline$G_{12}[\mathrm{GPa}]$ & 5 & $G_{13}[\mathrm{GPa}]$ & 5 & $G_{23}[\mathrm{GPa}]$ & 3.846 \\
\hline$\nu_{12}[-]$ & 0.27 & $\nu_{13}[-]$ & 0.27 & $\nu_{23}[-]$ & 0.3 \\
\hline$\rho\left[\mathrm{g} / \mathrm{cm}^{3}\right]$ & 1.58 & & & & \\
\hline
\end{tabular}

Table 1: Material properties of carbon fiber epoxy composite.

\section{Non-classical vibration modes}

In this section we report vibration modes observed in the conducted vibration analyses. These modes occurred as the first eigenmode. We focus 
on the influence of anisotropy due to geometry and hence the laminate layup is kept constant at $[0,90,90,0]$. Beside the expected out-of-plane modes we could also find in-plane and non-classical vibration modes due to the extremely high anisotropy and increased shear influence for large corrugation amplitudes.

Figure 4 shows the occurring mode shapes of the first mode as a function of the corrugation amplitude to periodic cell-length ratio $c / p$ and the aspect ratio $a / b$ for simply supported boundary conditions. For corrugation amplitudes lower or equal to 0.25 , which corresponds to a semi-circle, we observe out-of-plane modes. As the amplitude increases we find in-plane modes and for very large amplitudes and high aspect ratios a non-classical shear rotational mode. The mode shape of the non-classical shear rotational mode is illustrated for one unit-cell at the right hand side of the figure. The deformed unit-cell is a combination of a rotation about the $x$-axis at $z=0$ as well as an in-plane shear deformation along $y$.

Figure 4: Found mode shapes as a function of the corrugation amplitude and aspect ratio for simply-supported boundary conditions. The number of unit-cells is kept constant at a value of ten.

Figure 5 shows the occurring vibration modes for clamped boundary conditions as a function of the corrugation amplitude to periodic cell-length ratio $c / p$ and the aspect ratio $a / b$. For higher aspect ratios and corrugation amplitudes we observe in-plane vibration modes.

However, for the highest amplitude and aspect ratio we observe out-ofplane modes again. This can be explained with figure 6 . As the aspect 
Figure 5: Classification of found mode shapes as a function of the corrugation amplitude and aspect ratio for clamped boundary conditions. The number of unit-cells is kept constant at a value of ten.

ratio is increased from 1 to 2 as shown in a) and b), respectively, the tensile deformation becomes increasingly restricted due to the clamped boundary conditions and the mode changes from in-plane to out-of-plane. As we further increase the aspect ratio as shown in figure $6 \mathrm{c}$ ) we observe a non-classical counter-direction in-plane mode where mainly the parts on the neutral axis far away from the clamping deform.

Figure 6: Mode shapes for largest amplitude and clamped boundary conditions: a) $a / b=1$ b) $a / b=2$ c) $a / b=4$.

With increasing amplitudes the bending stiffness about the $y$-axis increases by several orders of magnitude while the axial stiffness in $y$-direction decreases by several orders of magnitude which explains the appearance of in-plane modes.

Characteristic examples of the deformed shapes are shown in the following. For corrugated panels with corrugation amplitudes corresponding to a semi-circle or smaller amplitudes we observe out-of-plane modes as illustrated in figure 7.

For very large amplitudes we observe in-plane modes as shown in figure 8 for a corrugated plate with simply-supported edges and an aspect ratio of one. 
Figure 7: Out-of-plane mode, the contour colors show the displacement in $z$-direction. All edges are clamped.

Figure 8: In-plane mode, the contour colors show the displacement in $y$-direction

As the aspect ratio is changed to a value of 2 , we observe for the same configuration a non-classical vibration mode (figure 9). In this mode we see both shear and rotational deformation and all unit-cells move in the same direction. The same deformed shape results if the width $b$ is further divided in half resulting in an aspect ratio of $a / b=4$.

Figure 9: Non-classical shear rotational mode, the contour colors show the displacement in $y$-direction

In the next example the aspect ratio is kept at 2, but the number of unit-cells and hence the length $a$ is increased by $50 \%$. We observe a nonclassical shear rotational mode where the unit-cells move in counter direction as illustrated in figure 10. If the width $b$ is divided in half resulting in an aspect ratio of $a / b=4$ we observe a shear rotational mode where all the unitcells move in one direction similar to the one shown in figure 9. If the number of unit-cells is further increased by a third and $b$ is kept constant $(a / b=2.67)$, we find a contour-direction shear rotational model. This indicates that the one-direction and counter-direction shear rotational modes might be in a similar range of deformation energy. 
Figure 10: Non-classical counter-direction shear rotational mode, the contour colors show the displacement in $y$-direction

We can summarize that we observe in-plane or non-classical vibration modes for very large amplitudes where we have ratios of $\tilde{D}_{11} / \tilde{D}_{22}$ in the range of $10^{8}$. For these bending stiffness ratios the aspect ratio seems to mainly influence the vibration behavior for simply supported boundary conditions. For quadratic corrugated panels, we observe an in-plane mode. As the aspect ratio increases the vibrations show a non-classical shear rotational eigenmode which can occur in form of a one-direction or counter-direction mode depending on the number of unit-cells and the aspect ratio. These modes cannot be modeled with classical equivalent plate models, since the influence of the mass placed away from the neutral axis, which influences the moment of inertia, as well as the increased influence of shear compliance are not considered.

\section{The valid solution space of homogenized models}

The results reported in the section before indicate that equivalent plate models have their limitations, when modeling vibration behavior of highly anisotropic corrugated laminates. While the in-plane modes can still be reproduced with classical plate models, the shear rotational modes require nonclassical models with higher complexity, such as Cosserat materials where the distribution of inertia is considered. In this paper we do not intend to develop such a Cosserat model, it should rather show non-classical effects and 
the validity of the solution space of homogenized models.

In the following section we compare the results of the detailed FEM model to the numerical equivalent plate model for different amplitudes and aspect ratios. Furthermore, we compare the two equivalent models, namely the numerical and the analytical to each other. The error between the values of the predicted first eigen frequency as well as the detected modes are investigated.

The relative error is calculated as follows:

$$
\operatorname{err}_{\text {rel }, i}=\frac{\left|f_{\text {Model }, i}-f_{\text {Model }, j}\right|}{f_{\text {Model }, i}}
$$

where $f_{M o d e l, i}$ and $f_{M o d e l, j}$ denote the eigen frequencies calculated with the respective models.

Figure 11 shows the comparison between the analytical and the numerical equivalent plate model for simply-supported boundary conditions as a function of aspect ratio $a / b$ and amplitude to periodic cell-length ratio $c / p$. The models agree very well with relative errors lower than $1 \%$. The models also predict the same mode shapes. The error tends to increase with decreasing aspect ratio. To obtain the numerical results for the in-plane modes the key option to only consider membrane element stiffness was activated in ANSYS.

Figure 11: Comparison between the numerical and analytical equivalent plate model for simply-supported boundary conditions

Figure 12 and 13 show the comparison between the detailed FEM model and the numerical equivalent plate model for simply-supported and clamped boundary conditions, respectively. For simply supported boundary conditions the model agrees very well for low amplitudes. Hence, for $c / p$-ratios 
lower than 0.5 the equivalent model is applicable for design purposes. Also for quadratic panels the errors are rather low. However, for $c / p$-ratios of 0.5 or higher and aspect ratios higher than 1, the comparison of the models show a high relative error. As the equivalent model assumes equally distributed mass it cannot predict non-classical effects. Further, in the equivalent model the shear stiffnesses are not precisely considered. In case of clamped boundary conditions the relative errors are in a similar range. Also in this case we observe increasing error with increasing amplitudes and aspect ratios. For low amplitudes and aspect ratios the results agree very well. The considered panels are all relatively small and hence the strong boundary conditions influence the result a lot. For high aspect ratios the results are sensitive to the shear stiffness $\tilde{K}_{44}$ which can explain the higher errors for these parameter configurations. However, for very large amplitudes and aspect ratios the equivalent model was not always able to predict the correct type of vibration mode.

Figure 12: Comparison between the detailed and the equivalent FE model for simplysupported boundary conditions

Figure 13: Comparison between the detailed and the equivalent FE model for clamped boundary conditions 


\section{Interaction of geometry and material anisotropy considering vi- bration behavior}

As already discussed the vibration behavior depends - besides boundary conditions and aspect ratio - on the anisotropy of the corrugated laminates. In this section we investigate the influence of anisotropy due to geometry and material on the vibration modes, since the equations used to calculate the equivalent stiffness properties in [12] suggest that these parameters are highly coupled. In particular different corrugation amplitudes, aspect ratios, and three different material lay-ups are studied: a $[0,0]_{s}^{\circ}$, a $[0,90]_{s}^{\circ}$, and a $[90,90]_{s}^{\circ}$. In this section the analytical model is used to calculate the vibration frequencies, hence, only the simply supported boundary conditions are considered. At the end of this section the influence of the material lay-up on the non-classical vibration modes is investigated.

While the out-of-plane modes depend on the $\tilde{D}$-matrix, the in-plane modes are influenced by the $\tilde{A}$-matrix. Figure 14 and 15 show the axial and bending stiffness values, respectively, as a function of material lay-up and corrugation amplitude. While the values of $\tilde{A}_{12}$ and $\tilde{D}_{11}$ are clearly

dominated by the change in the corrugation amplitude, the values $\tilde{A}_{11}$ and $\tilde{D}_{22}$ change by a good amount also with changing material lay-up. $\tilde{A}_{22}$ is influenced by both amplitude and lay-up. This makes it worth to investigate the influence of geometry and material on the vibration behavior.

Figure 14: Axial stiffness as a function of amplitude and material 
Figure 15: Bending stiffness as a function of amplitude and material

Looking at equation (5) we see that the out-of-plane natural eigen frequency is influenced by the bending stiffnesses by the following term:

$$
\left(\tilde{D}_{11}\left(\frac{a}{b}\right)^{4}+2\left(\tilde{D}_{12}+2 \tilde{D}_{66}\right)\left(\frac{a}{b}\right)^{2}+\tilde{D}_{22}\right)
$$

to which $\tilde{D}_{11}$ contributes the most, especially with increasing amplitude as we can see from figure 15. Hence, for aspect ratios of $a / b>1$ we don't expect a significant influence on the vibration behavior due to material changes. However, as the aspect ratio decreases the component $\tilde{D}_{22}$ becomes more important and the eigen frequency is significantly influenced by the material lay-up as indicated in figure 15. In particular, for aspect ratios that fulfill

$$
\left(\frac{a}{b}\right)^{4} \leq \frac{\tilde{D}_{22}}{\tilde{D}_{11}}
$$

the out-of-plane frequency is influenced by the anisotropy due to geometry and material and the vibration behavior can change. However, for configurations that do not fulfill conditions (12), only the anisotropy due to geometry has to be considered in order to analyze the out-of-plane vibration behavior. This is demonstrated in figure 16 where the first out-of-plane frequencies are plotted as a function of corrugation amplitude, aspect ratio, and material lay-up. For high aspect ratios $D_{11}$ is dominating the frequencies and the frequency only slightly changes with different material lay-ups. For very small aspect ratios, however, $D_{22}$ determines the value of the eigen frequency and the values are highly influenced by the material lay-up. 
Figure 16: Out-of-plane frequencies as a function of corrugation amplitude, material layup, and aspect ratio

Similar considerations can be made for the in-plane frequencies. Using equation (7) we see that with decreasing aspect ratio the influence of $\tilde{A}_{22}$ increases. Figure 17 shows the in-plane frequencies as a function of amplitude, aspect ratio, and material lay-up. While for high aspect ratios, the material lay-up does not influence the vibration frequencies, the influence becomes larger for low amplitudes.

Figure 17: In-plane frequencies as a function of corrugation amplitude, material lay-up, and aspect ratio

Figure 18 shows the occurring vibration modes as a function of corrugation amplitude, material lay-up, and aspect ratio. Apart from the findings for aspect ratios around 1 where we see in-plane modes for larger amplitudes, we can also observe that stiffer material lay-ups in $x$-direction show a higher tendency for in-plane modes. Furthermore, we can see that the aspect ratio has a large influence on the vibration mode. In case of very high aspect ratio we have an overall trend for in-plane modes, while for very low aspect ratios we only observe out-of-plane modes.

Figure 18: Vibration modes as a function of corrugation amplitude, material lay-up, and aspect ratio 
The above results are obtained with the analytical equivalent model which cannot represent non-classical deformation modes. Calculations with the detailed FE model show, however, that for high amplitudes and aspect ratios we observe non-classical modes. In particular, for the configuration with the same geometry as shown in figure 9 , we find with a $[0,0]_{s}^{\circ}$ lay-up a counterdirection shear rotational mode and for a $[90,90]_{s}^{\circ}$ lay-up a shear rotational mode where all unit-cells move in the same direction. This indicates that the material lay-up influences not only the eigen frequency, but also the occurring vibration mode. However, we have suggested in section 3 that these two modes are in a same range of deformation energy. As we increase the aspect ratio from 2 to 4 , we also observe shear rotational mode shapes. For the other configurations considered in section 3, we observe the same mode shapes for $[0,0]_{s}^{\circ}$ and $[90,90]_{s}^{\circ}$ lay-ups.

\section{Conclusion}

In this paper we investigated the vibration behavior of corrugated laminates using analytical and numerical methods. The high anisotropy due to geometry and material leads to in-plane as well as non-classical rotational vibration modes which are reported in this article. In a first step the vibration behavior due to geometry is explored using a detailed FEM shell model. For very high corrugation amplitudes we observe in-plane modes due to the very low compliance in in-plane direction as well as shear rotational modes that might occur due to inertia and shear effects that increase for higher amplitudes.

These findings are compared to equivalent models, namely a pre-integrated 
shell FE model and an analytical model using equivalent plate properties to represent the mechanical properties of the corrugated laminate. The equivalent models can only represent the classical modes of plate theory such as out-of-plane and in-plane modes. For these modes we could achieve a very good agreement between the equivalent model and the detailed FE model for low corrugation amplitudes and low aspect ratios. The shear rotational modes cannot be modeled with equivalent properties as long as these do not account for through-thickness material distribution. The inclusion of these effects in the equivalent model might be achieved with a Cosserat instead of a classical plate model. Also for larger amplitudes and aspect ratios the equivalent models cannot provide an accurate prediction of the eigen frequencies.

In a further step the complexity of the anisotropy is increased by superposing the geometrical anisotropy with material anisotropy. Analytical equations to determine the equivalent plate properties are combined with analytical formulations to calculate the out-of-plane and in-plane eigen frequencies. The results show that for high aspect ratios the eigen frequencies are mainly influenced by the anisotropy due to geometry. However, for very low aspect ratios the eigen frequencies depend also on the material anisotropy.

In a parametric study the eigenmodes of corrugated laminates are investigated concerning amplitude, material, and aspect ratio in order to determine the expected mode shapes.

The results show that for corrugated plates with very high anisotropy the limiting load case for design can change due to in-plane or non-classical vibration modes. Hence, the design of highly anisotropic corrugated laminates has to account for critical vibration modes. Furthermore, the anisotropy 
increases the stiffness in a specific direction, however, the hereby invoked compliance in the other direction can lead to new instabilities which do not occur in isotropic plates. In future studies we plan to develop enhanced plate models which take into account through-thickness material distribution such as Cosserat materials.

\section{Acknowledgment}

The authors gratefully acknowledge the support of the Swiss National Science Foundation (project no. 149200).

\section{References}

[1] I. Dayyani, a.D. Shaw, E. Saavedra Flores, M. Friswell, The Mechanics of Composite Corrugated Structures: A Review with Applications in Morphing Aircraft, Composite Structures 133 (2015) 358-380.

[2] E.-H. Hirschel, H. Prem, G. Madelung, J. W. Bergmann, Luftfahrtforschung in Deutschland, Bernard \& Graefe Verlag, 2001.

[3] M. R. M. Rejab, W. J. Cantwell, The mechanical behaviour of corrugated-core sandwich panels, Composites Part B: Engineering 47 (2013) 267-277.

[4] S. Kazemahvazi, D. Zenkert, Corrugated all-composite sandwich structures. Part 1: Modeling, Composites Science and Technology 69 (7-8) (2009) 913-919.

[5] C. Thill, J. Etches, I. Bond, K. Potter, P. Weaver, Morphing skins, Aeronautical Journal 112 (3216) (2008) 117-139. 
[6] T. Yokozeki, S.-i. Takeda, T. Ogasawara, T. Ishikawa, Mechanical properties of corrugated composites for candidate materials of flexible wing structures, Composites Part A: Applied Science and Manufacturing 37 (10) (2006) 1578-1586.

[7] C. Thill, J. a. Etches, I. P. Bond, K. D. Potter, P. M. Weaver, Composite corrugated structures for morphing wing skin applications, Smart Materials and Structures 19 (12) (2010) 124009. doi:10.1088/09641726/19/12/124009.

[8] S. Barbarino, O. Bilgen, R. M. Ajaj, M. I. Friswell, D. J. Inman, A Review of Morphing Aircraft, Journal of Intelligent Material Systems and Structures 22 (9) (2011) 823-877. doi:10.1177/1045389X11414084.

[9] F. Previtali, A. F. Arrieta, P. Ermanni, Performance of a ThreeDimensional Morphing Wing and Comparison with a Conventional Wing, AIAA Journal 52 (10) (2014) 2101-2113.

[10] Y. Xia, M. I. Friswell, Equivalent Models of Corrugated Laminates for Morphing Skins, Active and Passive Smart Structures and Integrated Systems 7977 (2011) 79771I-79771I-10. doi:10.1117/12.880433.

[11] Y. Xia, M. I. Friswell, E. I. S. Flores, Equivalent models of corrugated panels, International Journal of Solids and Structures 49 (13) (2012) $1453-1462$.

[12] G. Kress, M. Winkler, Corrugated laminate homogenization model, Composite Structures 92 (3) (2010) 795-810. 
[13] C. Thurnherr, L. Ruppen, G. Kress, P. Ermanni, Interlaminar stresses in corrugated laminates, Composite Structures 140 (2016) 296-308.

[14] G. Wang, S. Veeramani, N. M. Wereley, Analysis of Sandwich Plates with Isotropic Face Plates and a Viscoelastic Core, Journal of Vibration and Acoustics 122 (3) (2000) 305.

[15] J. Reddy, Theory and Analysis of Elastic Plates and Shells, second edi Edition, CRC Press, New York, 2007.

[16] N. Bardell, R. Langley, J. Dunsdon, On the free in-plane vibration of isotropic rectangular plates, Journal of Sound and Vibration 191 (3) (1996) 459-467.

[17] N. Semenyuk, I. Y. Babich, N. Zhukova, Natural vibrations of corrugated cylindrical shells, International Applied Mechanics 41 (5) (2005) 512519.

[18] B. Liu, Y. Xing, Comprehensive exact solutions for free in-plane vibrations of orthotropic rectangular plates, European Journal of Mechanics, A/Solids 30 (3) (2011) 383-395.

[19] R. M. Jones, Mechanics of Composite Materials, Hemisphere Publishing Corporation, New York, 1975.

[20] C. Thurnherr, Y. Mirabito, G. Kress, P. Ermanni, Highly anisotropic corrugated laminates deflection under uniform pressure, Composite Structures 154 (2016) 31-38. doi:10.1016/j.compstruct.2016.07.017. 


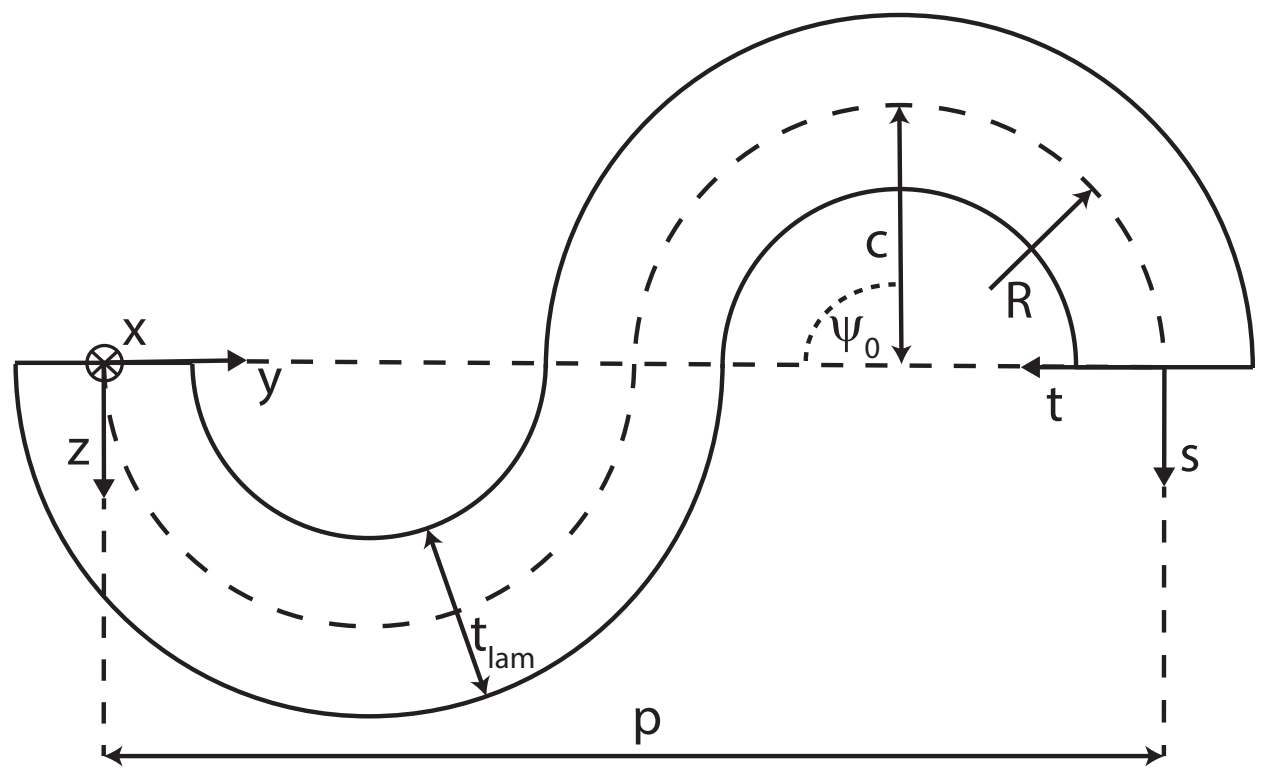

Figure 1: Definition of geometry of the corrugated structures 


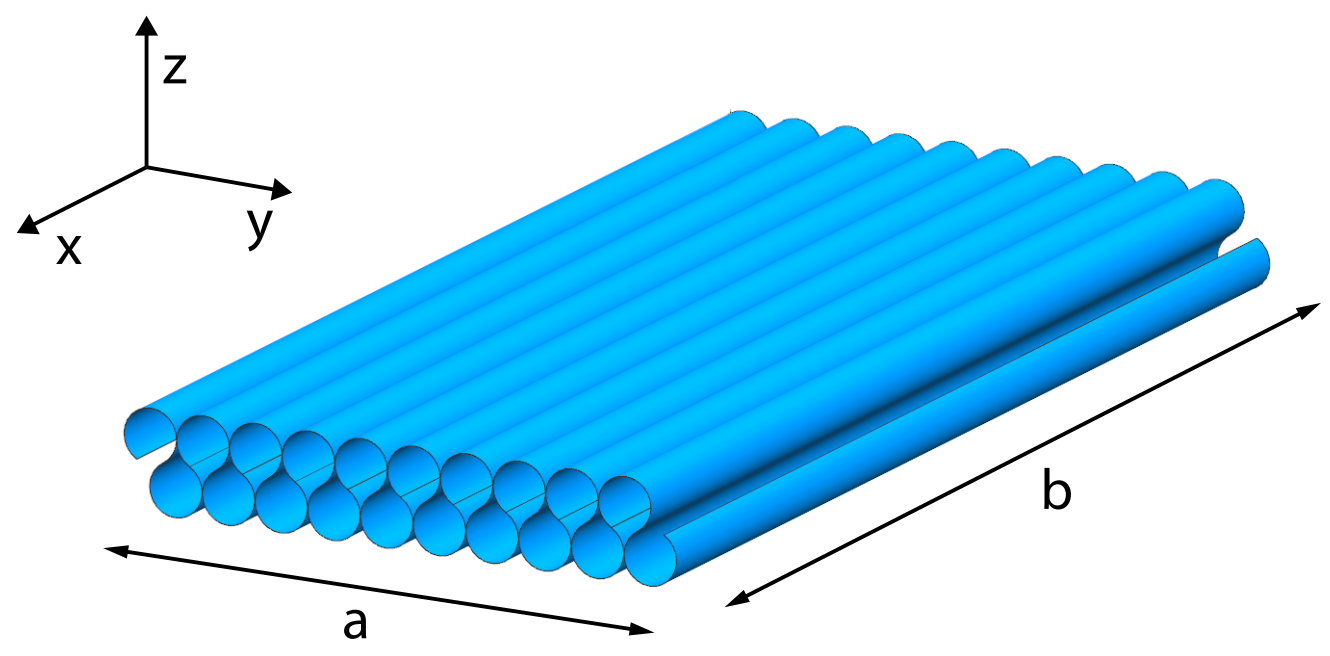

Figure 2: Definition of the corrugated plate geometry 


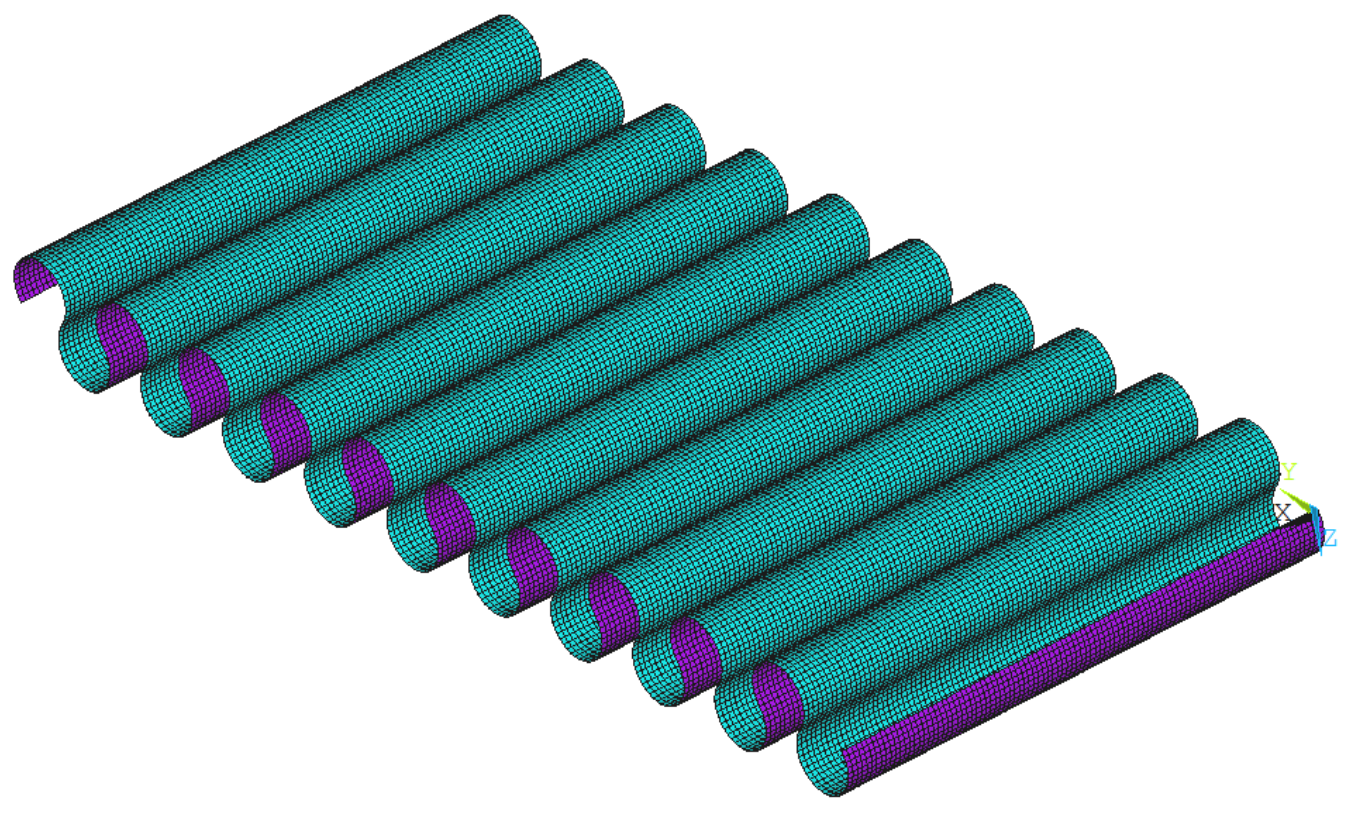

Figure 3: Detailed finite element shell model 


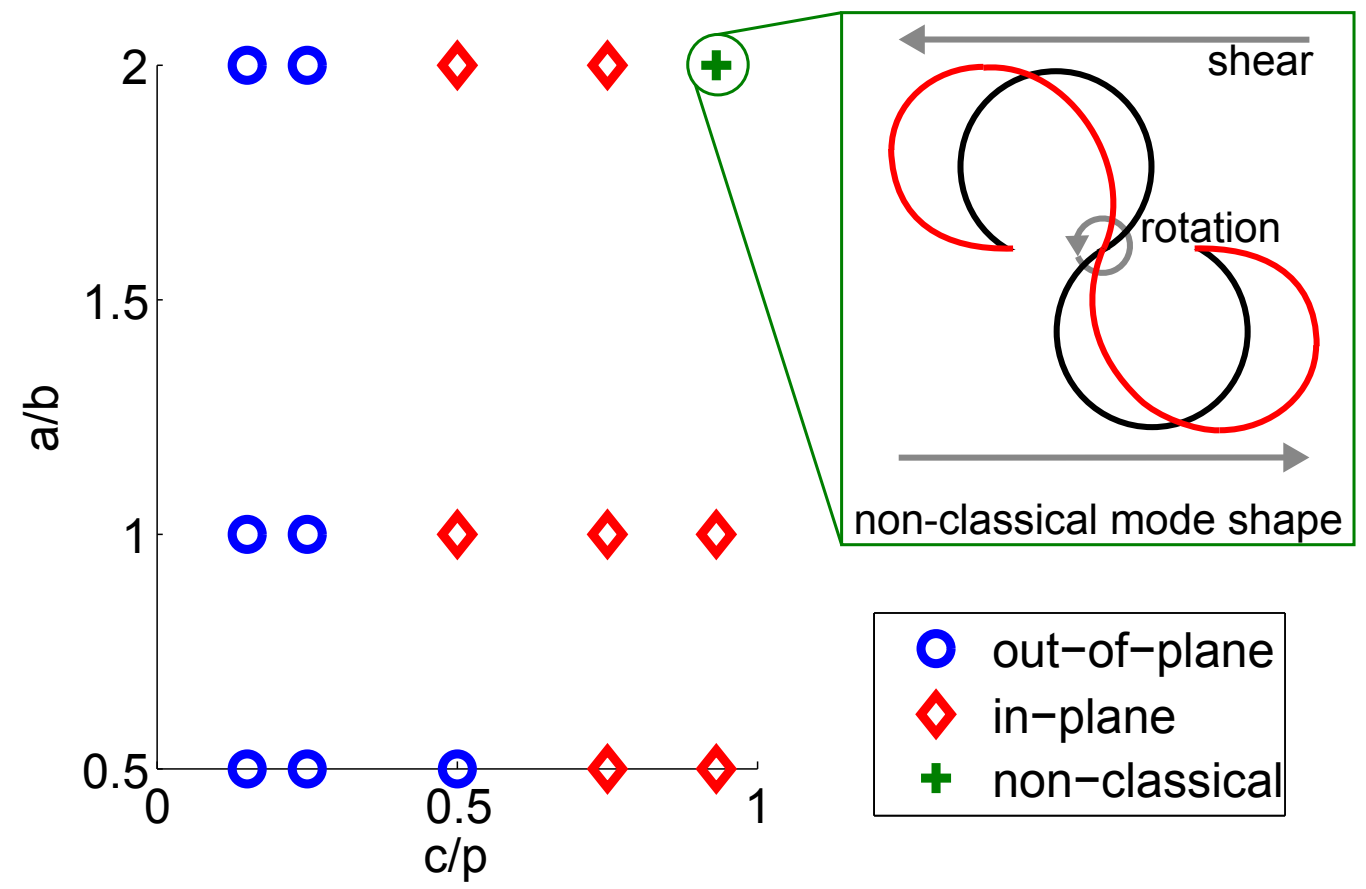

Figure 4: Found mode shapes as a function of the corrugation amplitude and aspect ratio for simply-supported boundary conditions. The number of unit-cells is kept constant at a value of ten. 


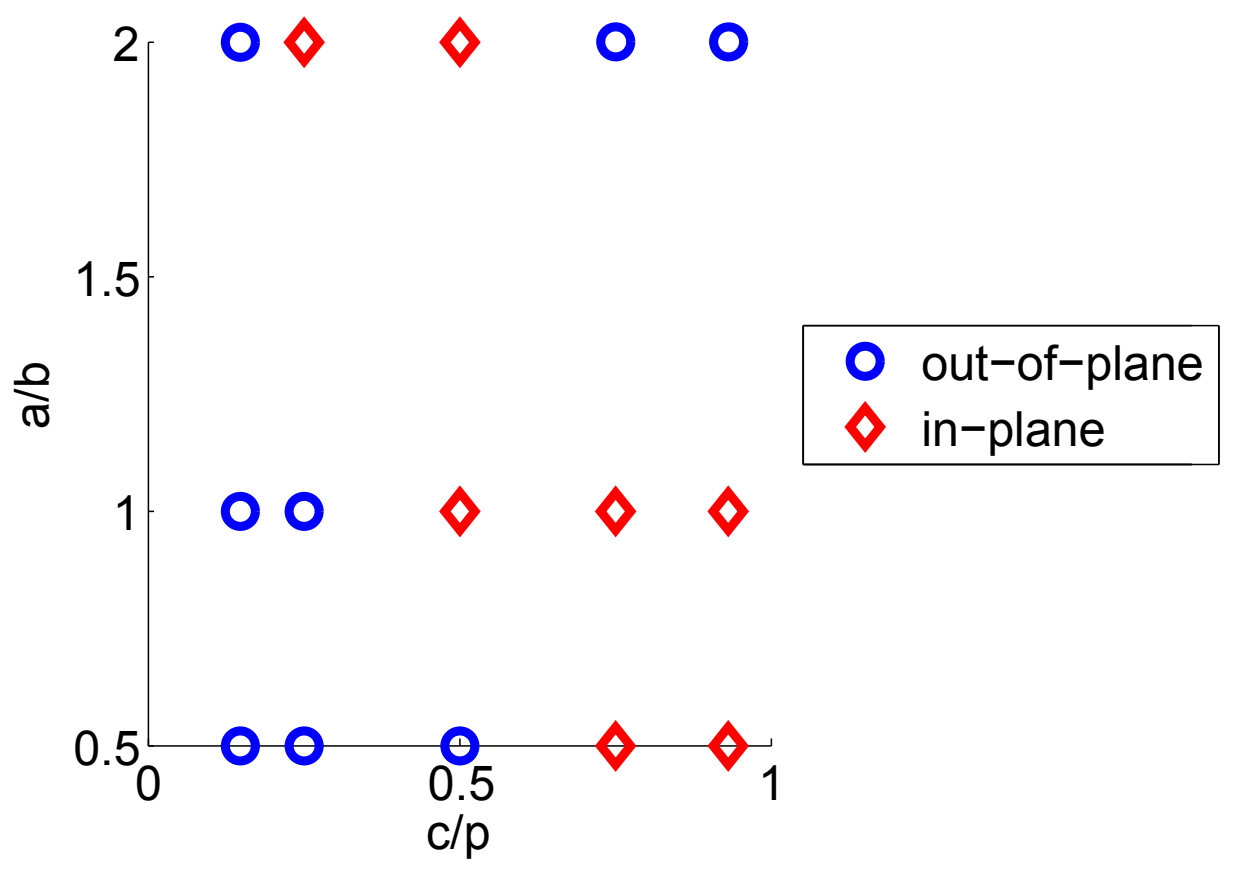

Figure 5: Classification of found mode shapes as a function of the corrugation amplitude and aspect ratio for clamped boundary conditions. The number of unit-cells is kept constant at a value of ten. 


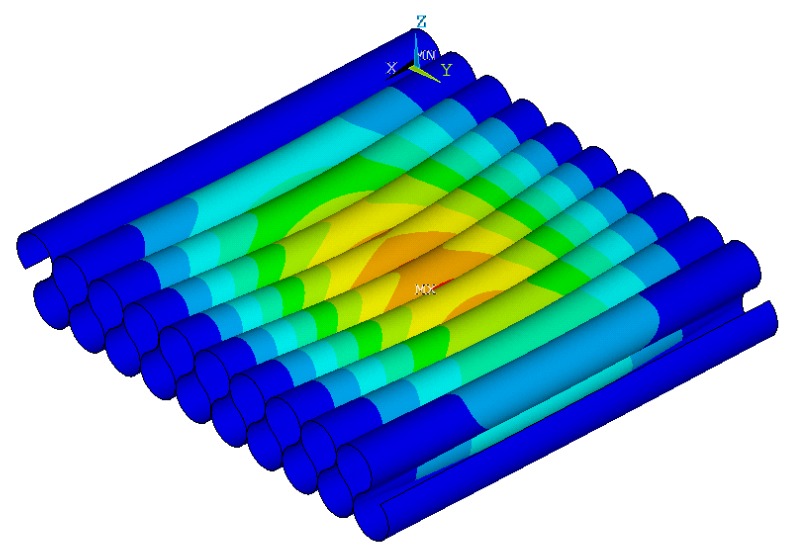

a) aspect ratio 1, contour colors show the displacement in in-plane y-direction

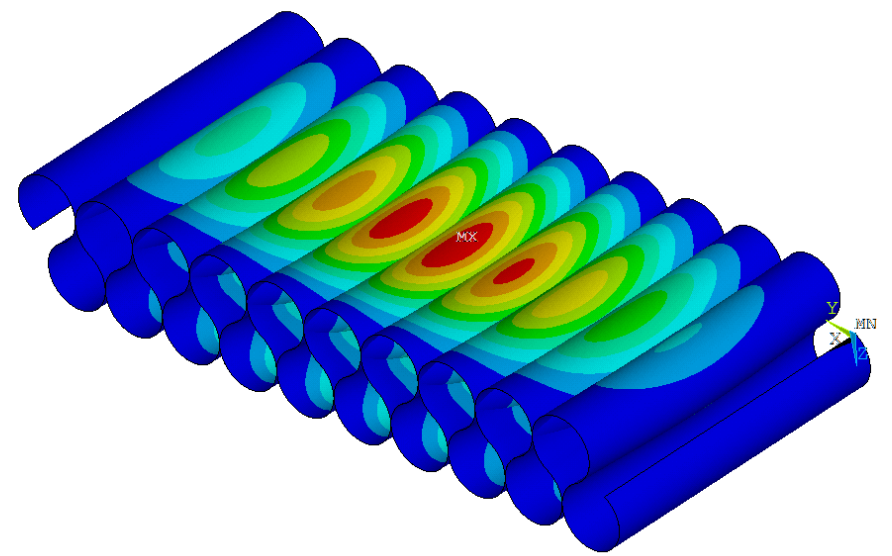

b) aspect ratio 2, contour colors show the displacement in out-of-plane z-direction

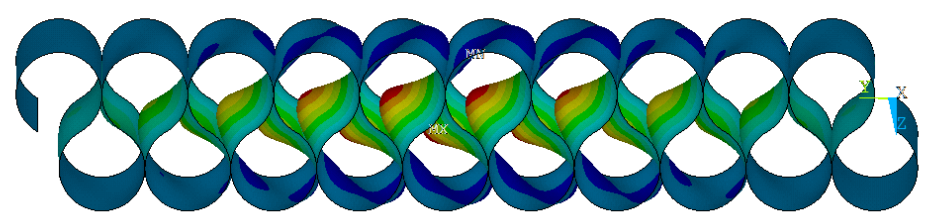

c) aspect ratio 4, contour colors show the displacement in in-plane y-direction

Figure 6: Mode shapes for largest amplitude and clamped boundary conditions: a) $a / b=1$ b) $a / b=2$ c) $a / b=4$. 


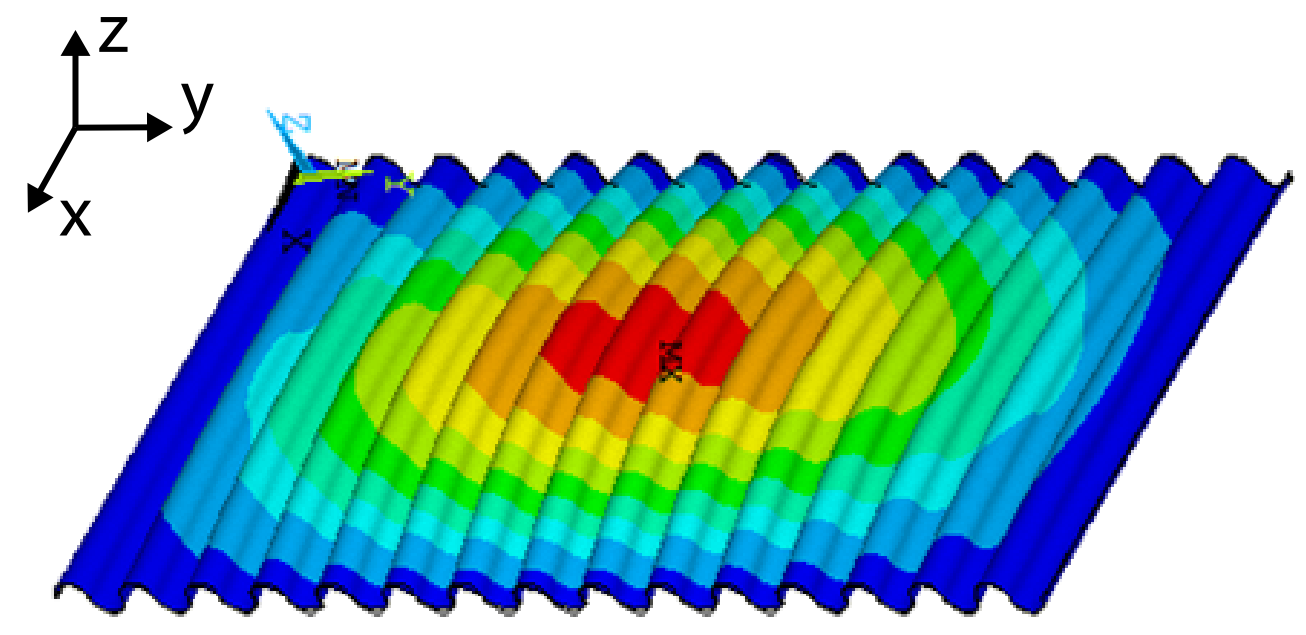

Figure 7: Out-of-plane mode, the contour colors show the displacement in $z$-direction. All edges are clamped. 


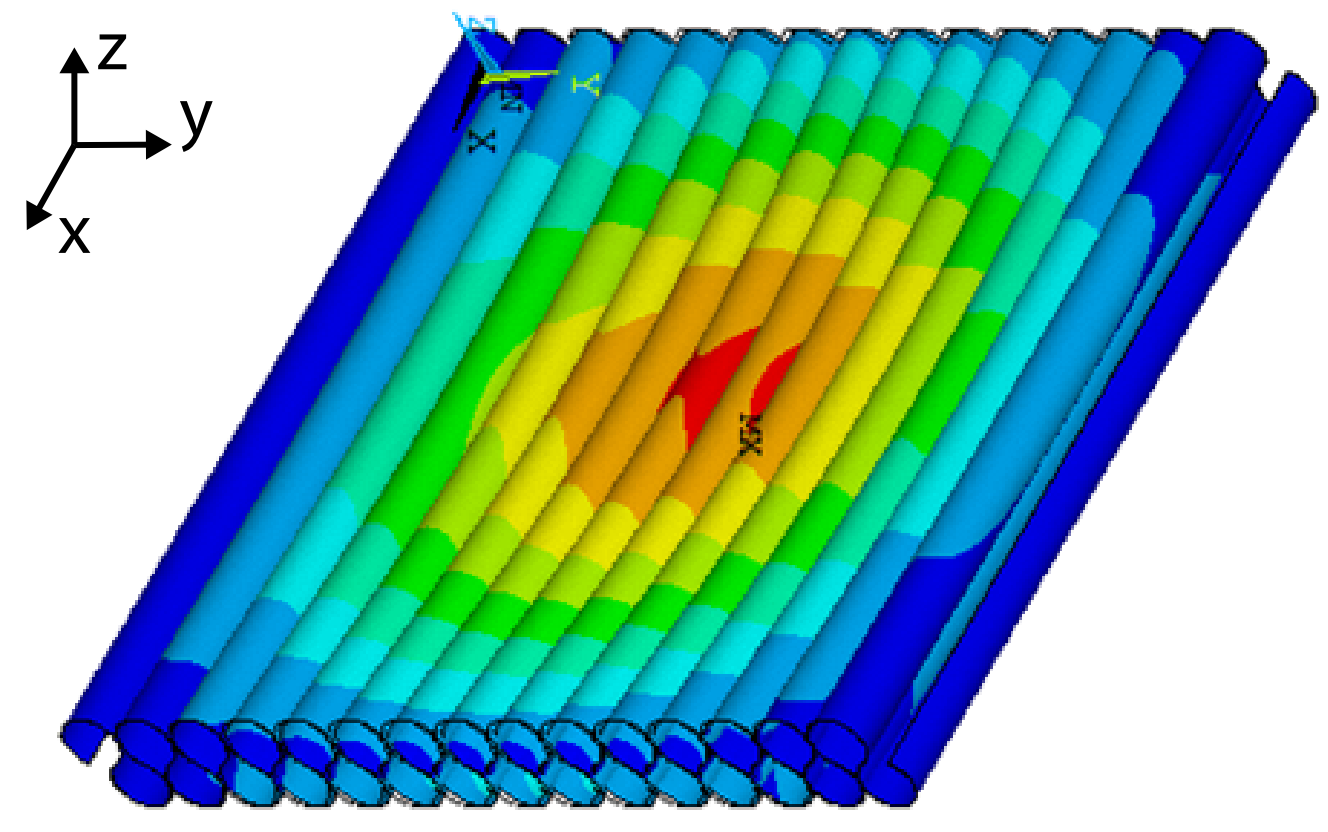

Figure 8: In-plane mode, the contour colors show the displacement in $y$-direction 

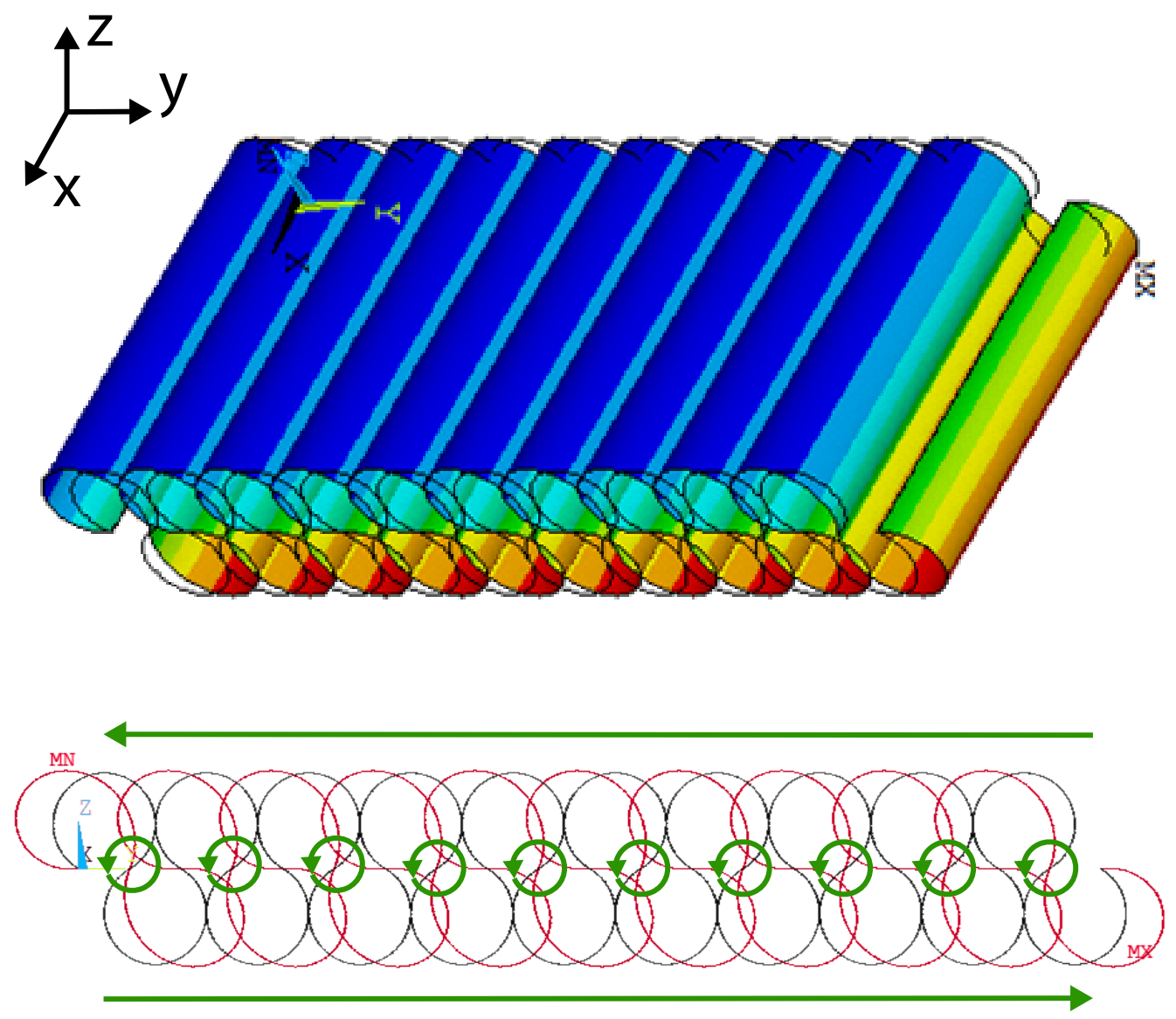

Figure 9: Non-classical shear rotational mode, the contour colors show the displacement in $y$-direction 

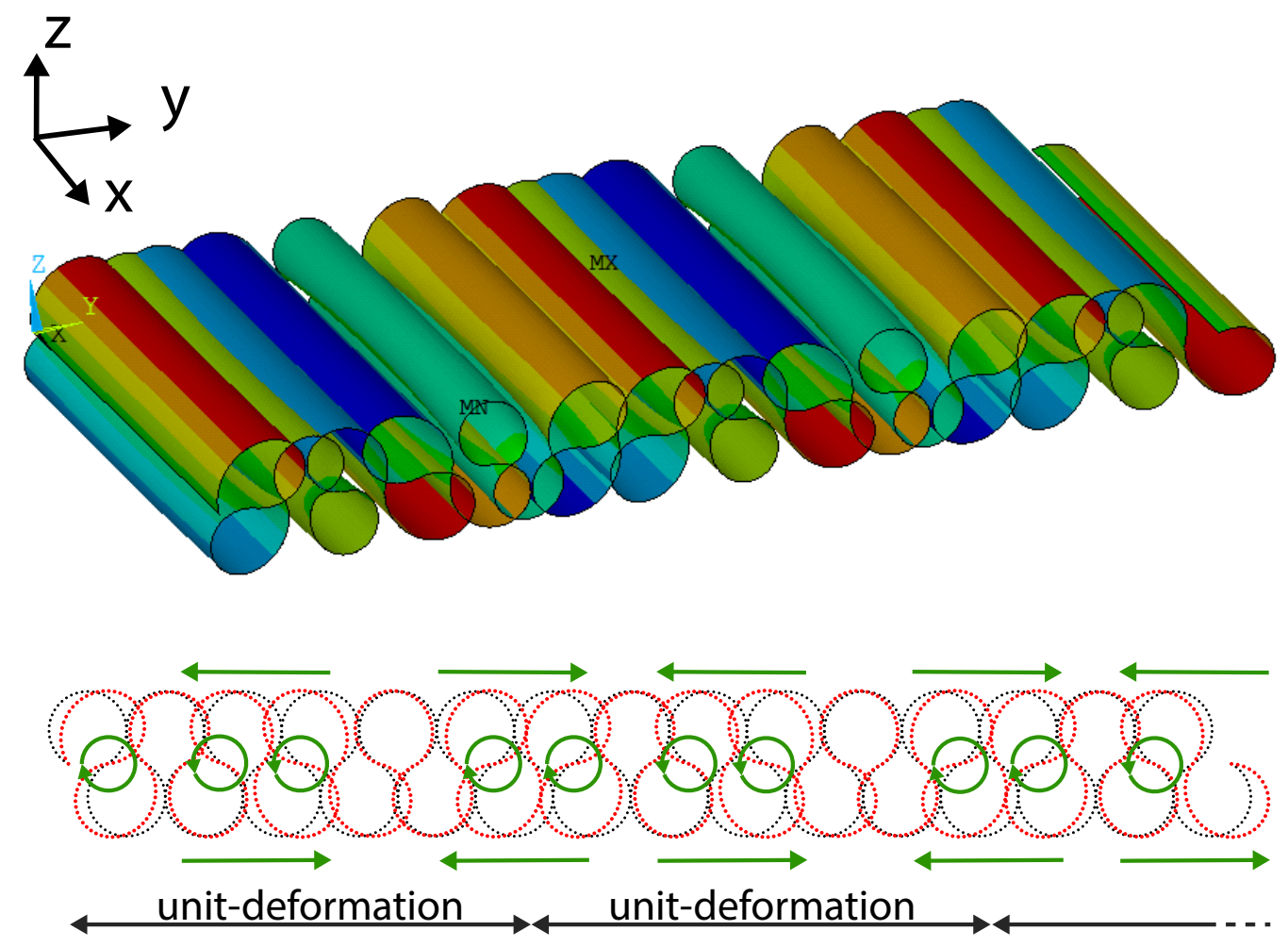

Figure 10: Non-classical counter-direction shear rotational mode, the contour colors show the displacement in $y$-direction 


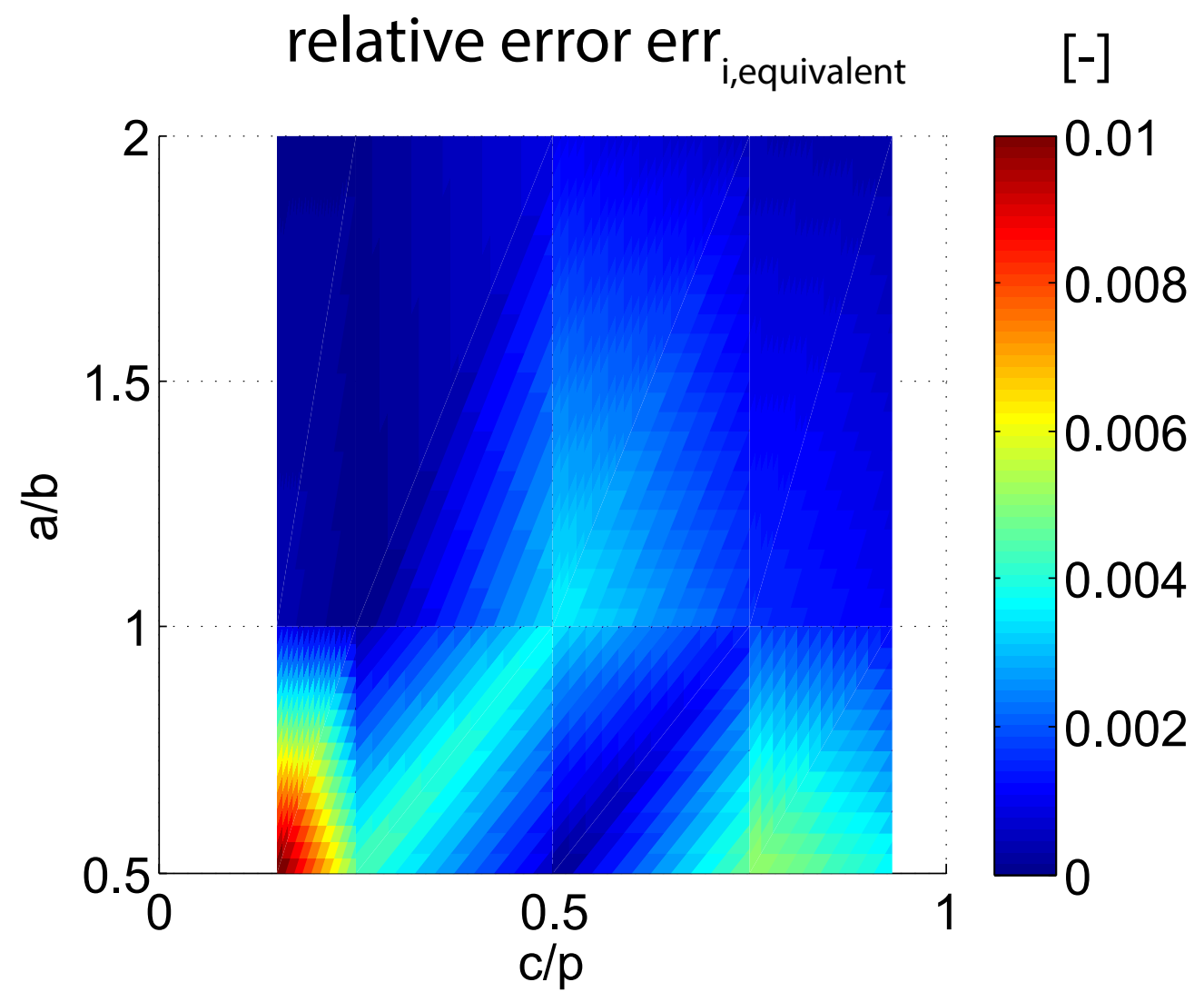

Figure 11: Comparison between the numerical and analytical equivalent plate model for simply-supported boundary conditions 


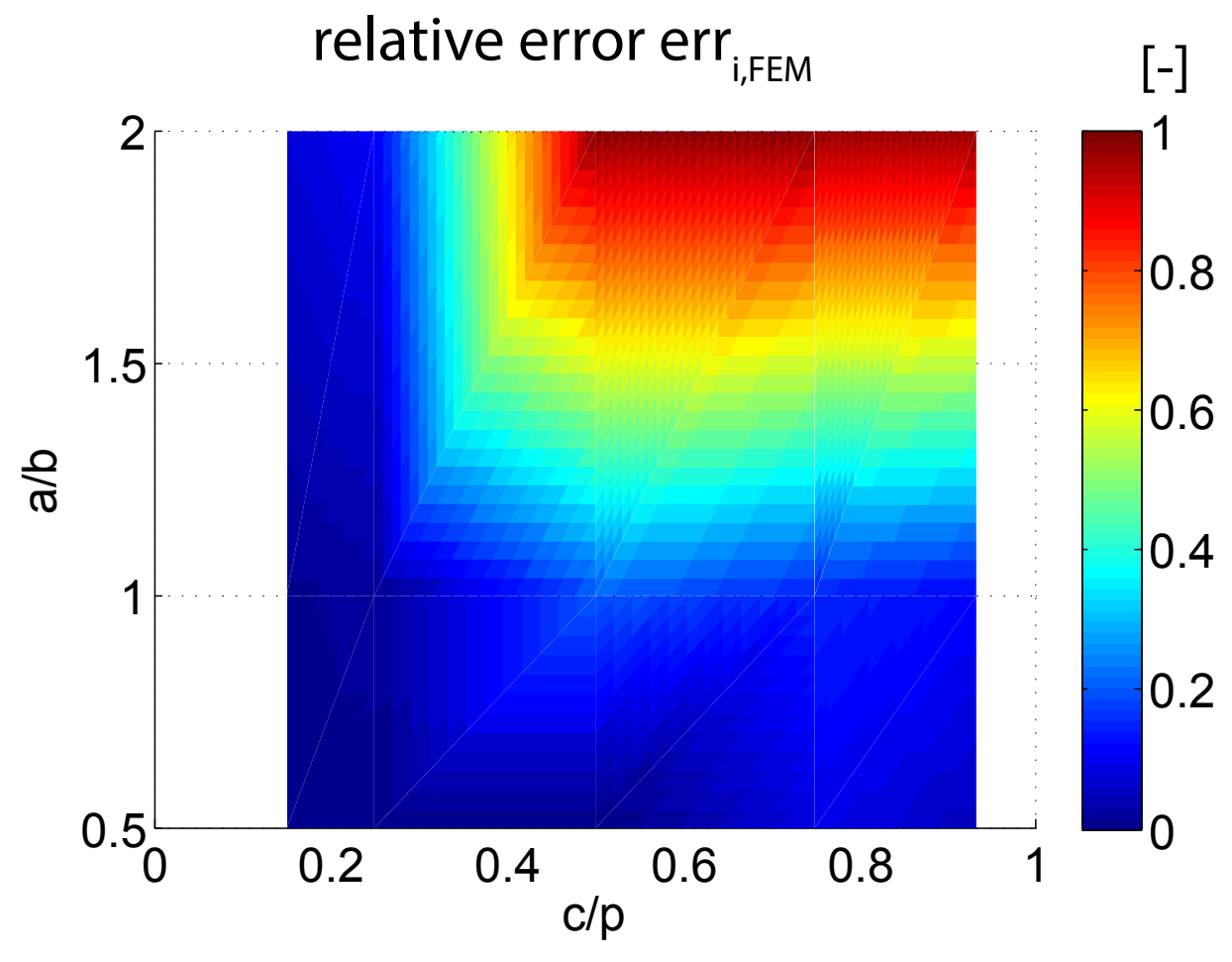

Figure 12: Comparison between the detailed and the equivalent FE model for simplysupported boundary conditions 


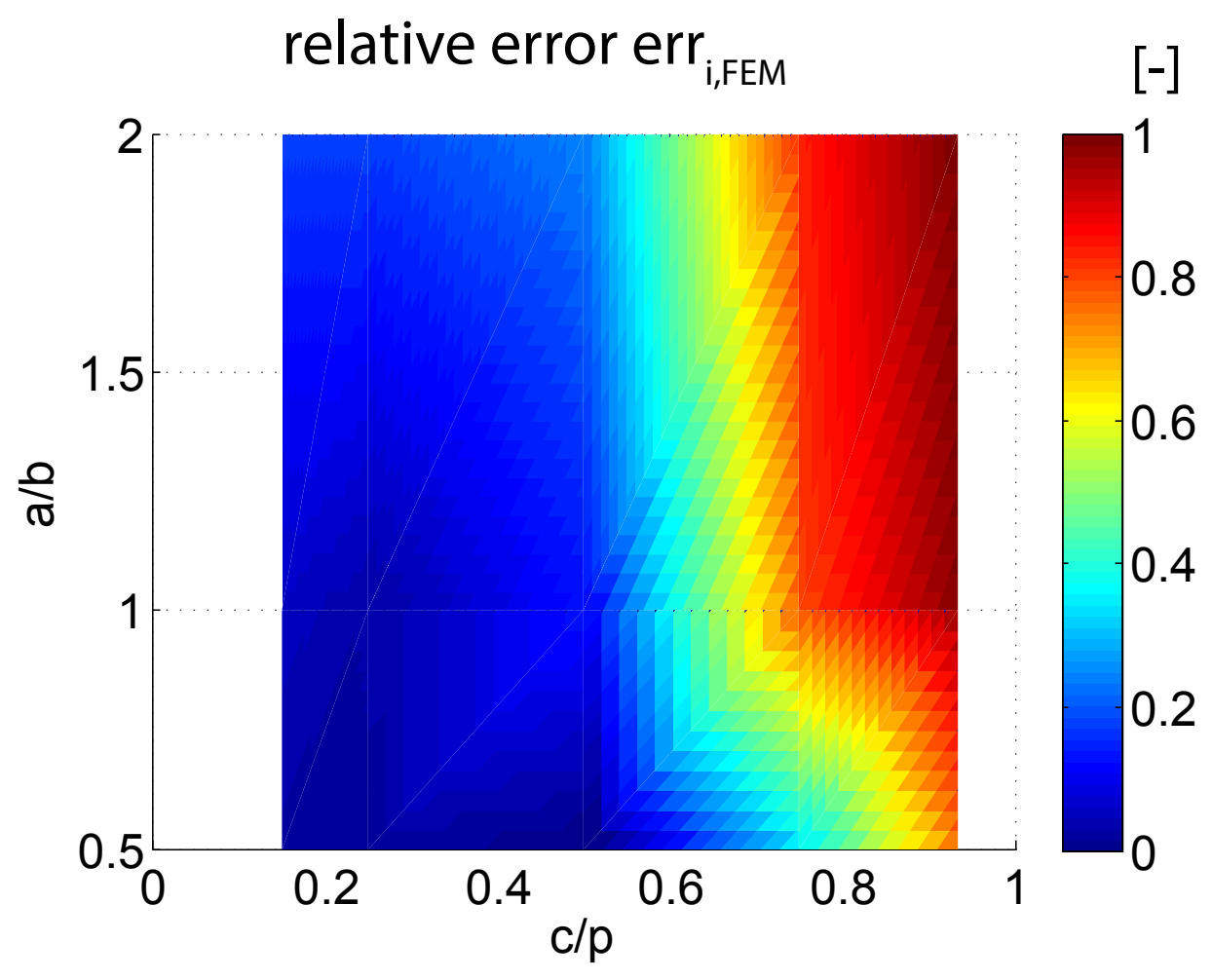

Figure 13: Comparison between the detailed and the equivalent FE model for clamped boundary conditions 


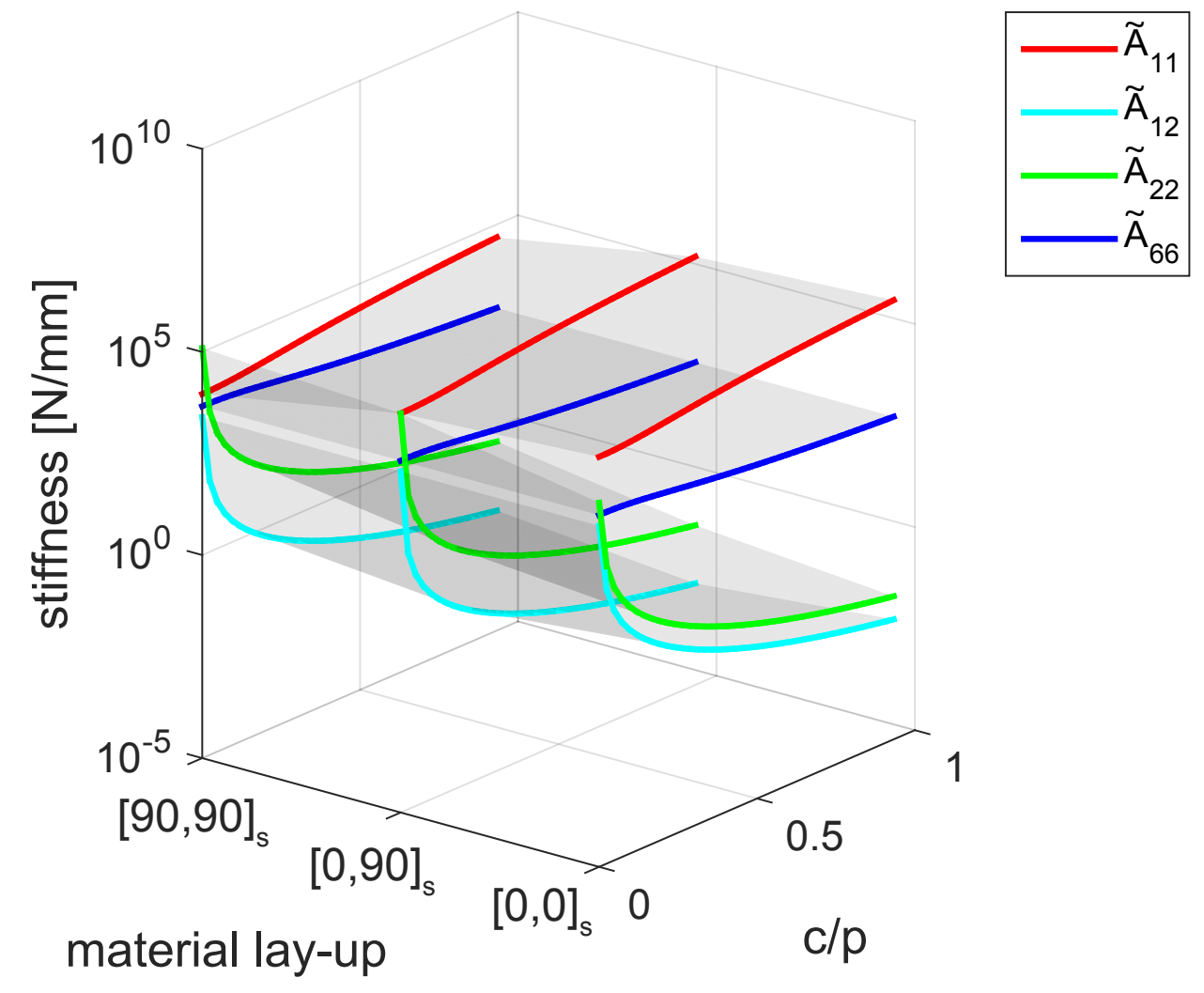

Figure 14: Axial stiffness as a function of amplitude and material 


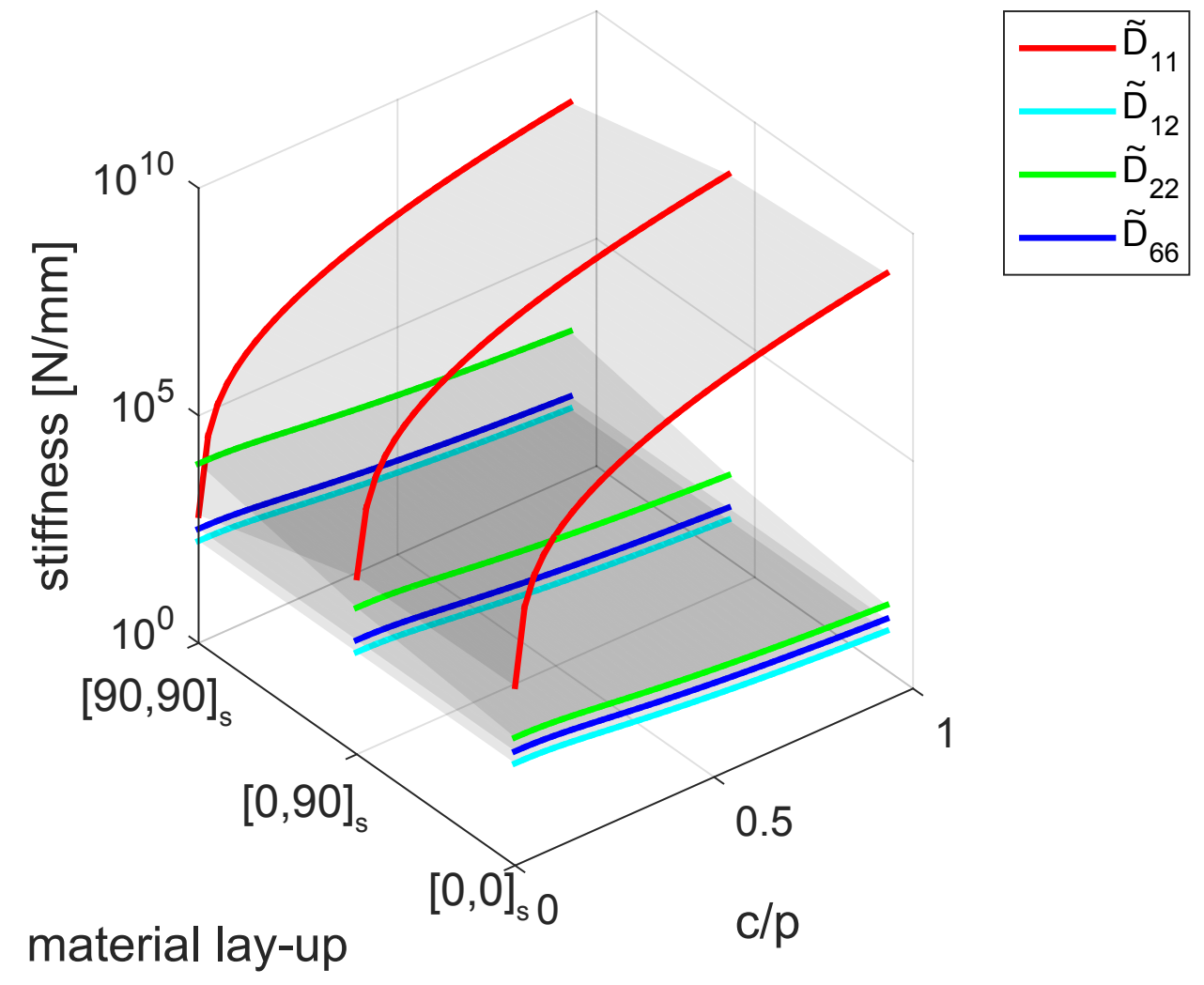

Figure 15: Bending stiffness as a function of amplitude and material 


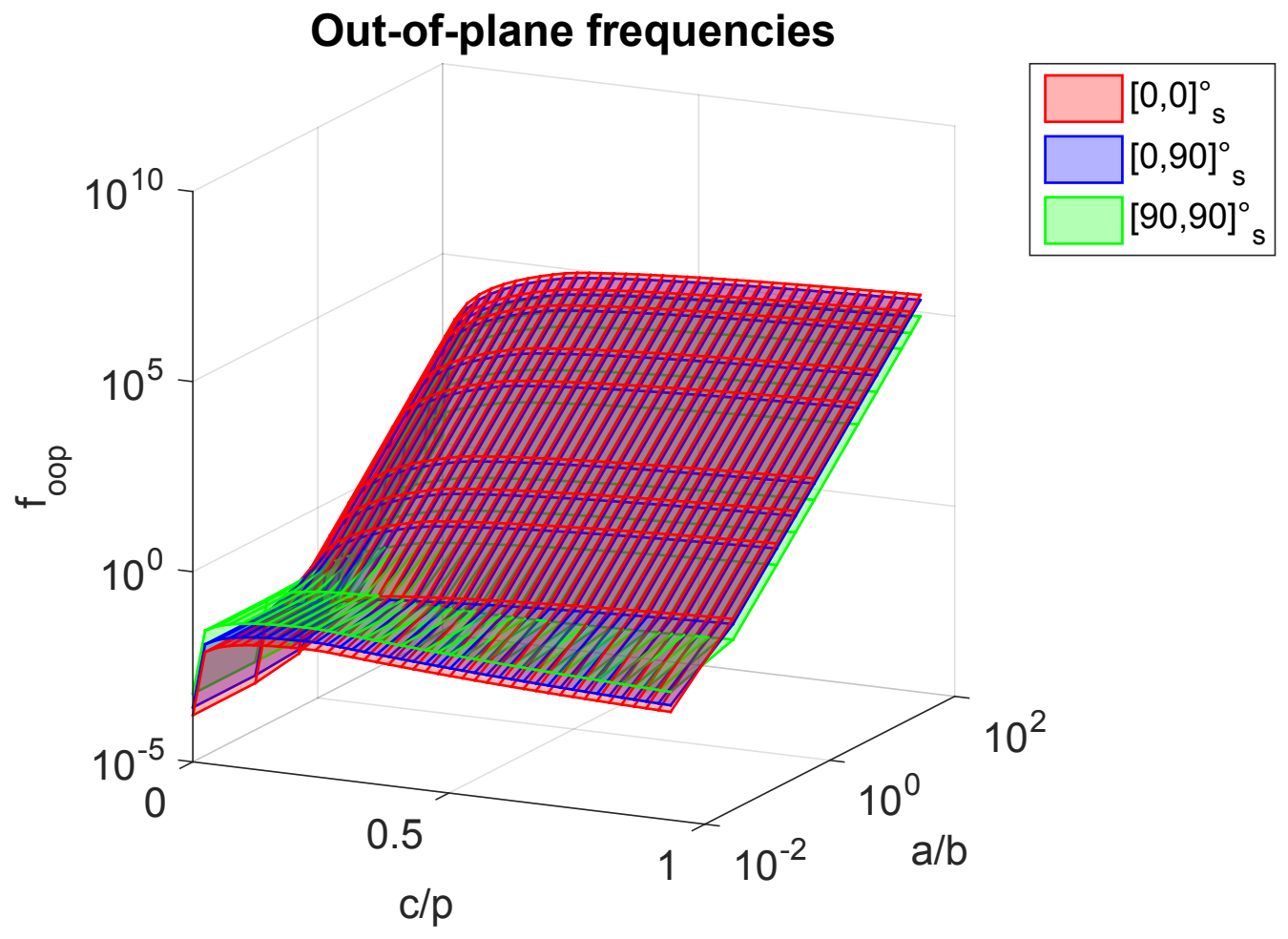

Figure 16: Out-of-plane frequencies as a function of corrugation amplitude, material layup, and aspect ratio 


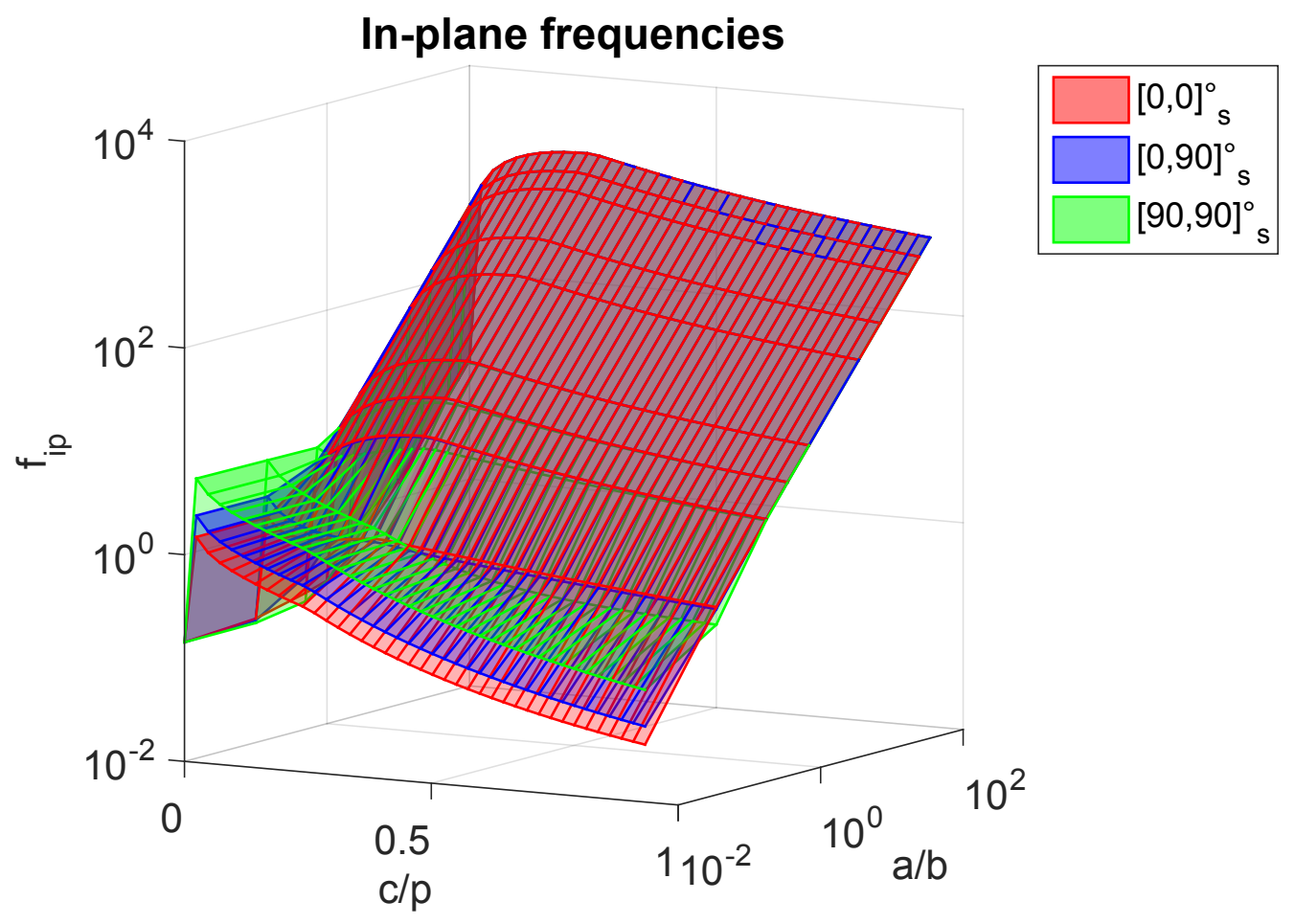

Figure 17: In-plane frequencies as a function of corrugation amplitude, material lay-up, and aspect ratio 


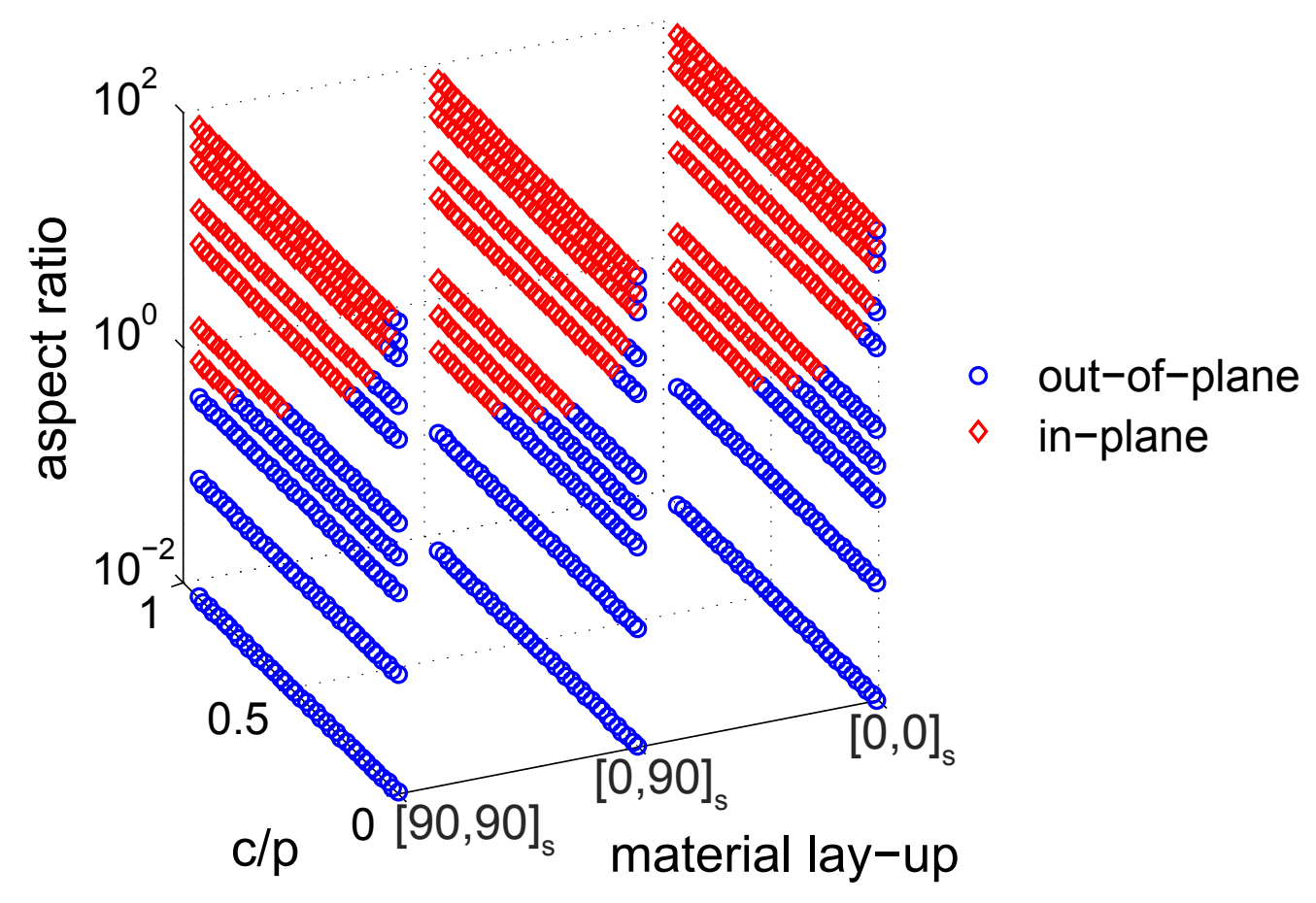

Figure 18: Vibration modes as a function of corrugation amplitude, material lay-up, and aspect ratio 